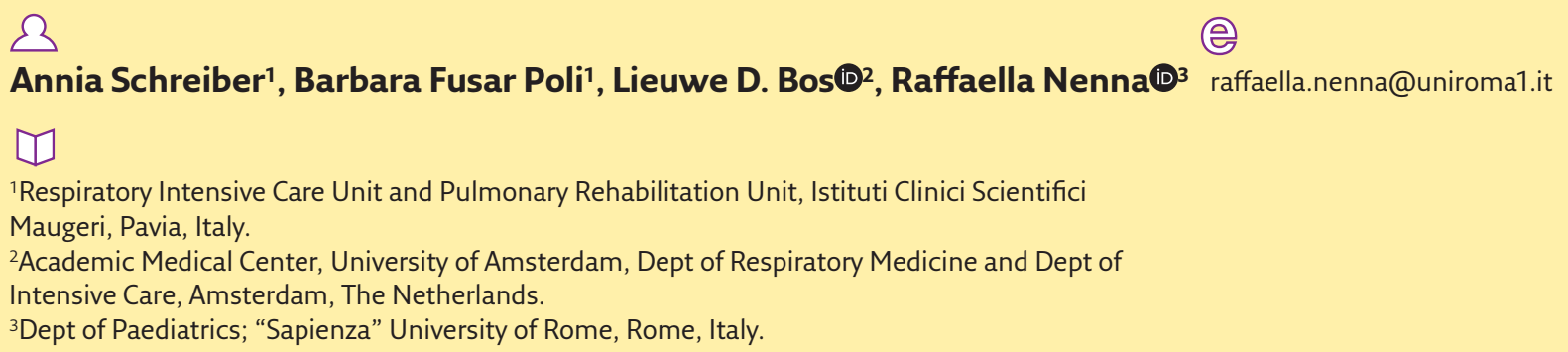

\title{
Noninvasive ventilation in
}

\section{hypercapnic respiratory failure: from rocking beds to fancy masks}

\section{Landmark papers in respiratory medicine}

Significant developments have occurred in noninvasive ventilation (NIV) over the past two to three decades, such that it is now one of the most evidence-based areas of respiratory medicine. NIV is now part of the standard of care for a variety of conditions such as hypercapnic respiratory failure due to acute exacerbation of chronic obstructive pulmonary disease (COPD) or cardiogenic pulmonary oedema[1, 2] and it has gained prominence in other settings such as in weaning from invasive mechanical ventilation (IMV), in the postoperative period and in palliative care [3]. Indications for the use of NIV continue to expand.

We are now used to seeing awake and cooperative patients with varying degrees of clinical severity being treated with ventilatory support administered via nasal or full-face masks, in both intensive care units (ICUs) and medical wards. Just 30 years ago, immediate intubation and treatment with IMV in the ICU setting was the only management option for patients presenting with severe acute hypercapnic respiratory failure. This evolution has brought not only a change in the mode of delivering ventilatory support but also in the related consequences and mortality rates.
In fact, the use of an artificial airway during IMV may give rise to a series of adverse effects. One of the most serious is that it predisposes patients to the development of pneumonia, by impairing cough and mucociliary clearance, and by promoting an accumulation of contaminated secretions above the cuff and subsequent leakage around the cuff itself $[4,5]$.

In this article, we attempt to present a chronological account of what we consider to have been some of the most significant research on NIV in acute hypercapnic respiratory failure over recent decades, determining a radical change or breakthrough in the approach to the various aetiologies of this condition.

A number of milestones were reached in the late 1980s/early 1990s, when several randomised controlled trials demonstrated that NIV could achieve the same physiological response [6, 7] and rapid improvement in gas exchange as conventional mechanical ventilation in a number of clinical conditions, with the huge advantage of not being associated with the same complications, length of hospital stay and risk of death [8-10].
Cite as: Schreiber $A$, Fusar Poli B, Bos LD, et al. Noninvasive ventilation in hypercapnic respiratory failure: from rocking beds to fancy masks. Breathe 2018; 14: 235-237. 


\section{Physiological response and parameters predicting NIV success}

In 1989 and 1991, Meduri et al. [6, 7] conducted two preliminary studies in a heterogeneous group of patients with acute respiratory failure (ARF) due to intrinsic lung disease. In both studies, the aim was to assess the feasibility of noninvasive treatment in patients who would normally have required conventional mechanical ventilation and to identify the parameters of physiological responses to NIV. In the second study [7], a continuation of the first one on a larger scale, the aim was also extended to identify early predictors of NIV success. In both studies, face mask ventilation was proven to be feasible, and effective in correcting gas exchange abnormalities and avoiding intubation in almost $70 \%$ of patients, despite the severe underlying lung impairment.

Regarding predictors of success, within the first hours after starting NIV a "successful" and a "failure" patient group emerged. The "successful group" was characterised by a significant and progressive drop in mean carbon dioxide tension $\left(P \mathrm{CO}_{2}\right)$, a rise in $\mathrm{pH}$ and a decrease in respiratory rate at 1 and 2-6 h after onset of NIV. In the "failure group" (which eventually required re-intubation), after an initial nonsignificant improvement, mean $\mathrm{PCO}_{2}$, respiratory rate and $\mathrm{pH}$ returned to values close to baseline or failed to improve further. The authors concluded that significant improvements in $\mathrm{PCO}_{2}(>16 \%$ decrease at $1 \mathrm{~h}$ ) and pH (from $<7.30$ to $>7.30$ at the 2-6-h interval; positive predictive value of $92 \%$ ) after the first few hours of NIV identified patients who could be successfully treated with short-term NIV. By contrast, a successful response to treatment and NIV duration could not be predicted by the severity of the underlying lung disease or by the $\mathrm{PCO}_{2}$ and $\mathrm{pH}$ values obtained before initiating ventilation. In both studies, the delay in intubating patients who failed the NIV treatment trial was not associated with complications.

Even if these relatively small preliminary trials were not randomised and controlled, they have the merit of having demonstrated the similarity between NIV and conventionally delivered ventilation in terms of physiological response, and of having suggested that NIV is a safe and valuable option in patients with acute hypercapnic respiratory failure.

\section{NIV in acute exacerbation of COPD with respiratory acidosis (versus standard treatment)}

A few years later, BROCHARD et al. [8] conducted a multicentre, prospective, randomised trial in 85 COPD patients admitted to five ICUs with acute exacerbations, to compare the efficacy of face mask
NIV (pressure support set initially at $20 \mathrm{cmH}_{2} \mathrm{O}$, used for at least $6 \mathrm{~h}$ per day) plus standard medical treatment to standard medical treatment alone. Only 11 (26\%) out of the 43 patients in the NIV group required endotracheal intubation, as compared to 31 (74\%) out of the 42 patients in the standard medical treatment group $(p<0.001)$. The hospital stay was also significantly shortened $(23 \pm 17$ versus $35 \pm 33$ days; $p<0.02$ ) and mortality significantly reduced ( $9 \%$ versus $29 \%$; $p<0.02$ ) with the use of NIV.

In this study, the authors demonstrated that NIV was able both to reduce the proportion of COPD patients with ARF needing IMV and to improve their outcome (by reducing the associated complications, mortality and length of hospitalisation). However, careful selection of patients was a crucial point. In fact, only $29 \%$ of patients admitted were eligible for NIV as the authors included patients who were likely to need endotracheal intubation, but excluded those needing immediate intubation and those with a clear cause of decompensation requiring a specific therapeutic approach, such as heart failure, pneumonia or sepsis.

We consider this study a milestone in the field because, at the time of its publication, IMV was still the preferred mode of ventilation for COPD patients with ARF, and it was the first study to establish the indication for NIV in COPD exacerbations with respiratory acidosis, contributing to making it a standard of care in this specific condition.

\section{NIV in weaning (versus continuation of conventional mechanical ventilation)}

In 1998, in another multicentre, randomised trial, Nava et al. [9] became the first to use NIV to facilitate weaning from IMV, experimenting with a new weaning approach. The aim of the study was to determine whether NIV could improve the outcome of weaning in difficult-to-wean COPD patients.

After $24-48 \mathrm{~h}$ of conventional ventilation delivered through an endotracheal tube, a T-piece trial was started in all patients. At T-piece failure, 25 patients were randomly assigned to be extubated and underwent the weaning process with NIV, while 25 remained intubated and continued to receive invasive pressure support ventilation.

As compared with patients who were reconnected to IMV, patients who were weaned using the noninvasive technique had a significantly higher weaning success rate $(\mathrm{p}=0.002)$, spent significantly fewer days receiving mechanical ventilation ( $16.6 \pm 11.8$ days versus $10.2 \pm 6.8$ days, respectively; $\mathrm{p}=0.021)$ and in the ICU $(24.0 \pm 13.7$ days versus $15.1 \pm 5.4$ days, respectively; $\mathrm{p}=0.005$ ). Furthermore, the incidence of complications, including nosocomial pneumonia (no patients in the NIV group versus $28 \%$ in the IMV group), was 
different and, accordingly, 60-day mortality was significantly lower in the NIV group (72\% versus $92 \%$ in the IMV group; $p=0.009$ ).

Even if the trial involved only selected intubated COPD patients with difficult weaning, it was the first to show the usefulness of NIV as a tool for early extubation and to demonstrate the role of NIV in preventing re-intubation in the weaning phase.

\section{NIV after extubation (versus standard oxygen)}

The final randomised controlled trial we would like to mention was conducted in three ICUs in Spain and published some years later with the aim of assessing the effectiveness of NIV as compared with conventional oxygen in patients who underwent planned extubation [10].

A total of 106 mechanically ventilated patients with chronic respiratory disorders and hypercapnia were enrolled following a successful spontaneous breathing trial. 54 of them underwent continuous NIV (mean levels of inspiratory and expiratory positive-airway pressure were $17 \mathrm{cmH}_{2} \mathrm{O}$ and $6 \mathrm{cmH}_{2} \mathrm{O}$, respectively) for $24 \mathrm{~h}$ and 52 patients received conventional Venturi oxygen treatment (the control group).

Respiratory failure arose in significantly fewer patients in the NIV group as compared with the control group (15\% versus 48\%; $p<0.0001$ ) and 90 -day mortality was significantly lower $(11 \%$ in the NIV group as compared with $31 \%$ in control group; $p=0.024)$. A collateral finding was that about two-thirds of the patients who developed respiratory failure, but who did not need immediate reintubation, benefited from rescue therapy with NIV without resulting in delayed reintubation.

In our view, the valuable contribution of this study was two-fold: 1) it demonstrated the benefits of the early use of NIV in lessening the likelihood of respiratory failure after extubation in high-risk patients; and 2) it validated rescue therapy with NIV as a useful alternative to reintubation in patients who developed respiratory failure without major criteria for reintubation.

\section{Future directions}

The progressive replacement of IMV with NIV in a growing number of clinical conditions, and its proven effectiveness and consequent increasing use in different settings, has avoided a number of routine complications related to IMV and brought about a series of significant improvements, mainly in terms of length of hospital stay and mortality, and sedation and related improvements in quality of life.

However, there are still several challenges facing researchers in this field: some data still await solid confirmation and certain aspects of NIV remain controversial. In particular, the best noninvasive approach to hypoxaemic respiratory failure, the optimal strategy and monitoring technique to improve patient-ventilator synchrony, and the provision of more comfortable and efficient interfaces remain crucial issues to be solved.

However, it is the search for solutions to complex problems that brings about evolution and progress in every field. Therefore, we can only hope that we continue to have critical issues to overcome in this field if we aim to conduct forward-thinking clinical research and improve the lives of our patients.

\section{Conflict of interest}

None declared.

\section{References}

1. Nava S, Hill N. Non-invasive ventilation in acute respiratory failure. Lancet 2009; 374: 250-259.

2. Vital FMR, Ladeira MT, Atallah AN. Non-invasive positive pressure ventilation (CPAP or bilevel NPPV) for cardiogenic pulmonary oedema. Cochrane Database Syst Rev. 2013; 5: CD005351.

3. Rochwerg B, Brochard L, Elliott MW, et al. Official ERS/ATS clinical practice guidelines: noninvasive ventilation for acute respiratory failure. Eur Respir J 2017; 50: 1602426.

4. Stauffer JL, Olson DE, Petty TL. Complications and consequences of endotracheal intubation and tracheotomy: a prospective study of 150 critically ill adult patients. Am J Med 1981; 70: 65-76.

5. Fagon JY, Chastre J, Hance AJ, et al. Nosocomial pneumonia in ventilated patients: a cohort study evaluating attributable mortality and hospital stay. Am J Med 1993; 94 : $281-288$
6. Meduri GU, Conoscenti CC, Menashe PH, et al. Noninvasive face mask ventilation in patients with acute respiratory failure. Chest 1989; 95: 865-870.

7. Meduri GU, Abou-Shala N, Fox RC, et al. Noninvasive face mask ventilation in patients with acute respiratory failure. Chest 1991; 100: 445-454.

8. Brochard L, Mancebo J, Wysocki M, et al. Noninvasive ventilation for acute exacerbations of chronic obstructive pulmonary disease. N Engl J Med 1995; 333: 817-822.

9. Nava S, Ambrosino N, Clini E, et al. Noninvasive mechanical ventilation in the weaning of patients with respiratory failure due to chronic obstructive pulmonary disease. A randomized, controlled trial. Ann Intern Med 1998; 128: 721-728.

10. Ferrer $M$, Sellarés J, Valencia $M$, et al. Non-invasive ventilation after extubation in hypercapnic patients with chronic respiratory disorders: randomised controlled trial. Lancet 2009; 374: 1082-1088. 